\title{
High-Precision Propagation-Loss Measurement of Single-Mode Optical Waveguides on Lithium Niobate on Insulator
}

\author{
Jintian Lin ${ }^{1,+}$, Junxia Zhou ${ }^{2,3,+}$, Rongbo Wu ${ }^{1,4}$, Min Wang ${ }^{2,3}{ }^{\oplus}$, Zhiwei Fang ${ }^{2,3}$, Wei Chu ${ }^{1}$, \\ Jianhao Zhang ${ }^{1,4}$, Lingling Qiao ${ }^{1}$ and Ya Cheng ${ }^{1,2,3,4,5, * \mathbb{D}}$ \\ 1 State Key Laboratory of High Field Laser Physics, Shanghai Institute of Optics and Fine Mechanics, \\ Chinese Academy of Sciences, Shanghai 201800, China; jintianlin@siom.ac.cn (J.L.); rbwu@siom.ac.cn (R.W.); \\ chuwei@siom.ac.cn (W.C.); jhzhang@siom.ac.cn (J.Z.); qiaolingling@siom.ac.cn (L.Q.) \\ 2 State Key Laboratory of Precision Spectroscopy, East China Normal University, Shanghai 200062, China; \\ 5218092020026@stu.ecnu.edu.cn (J.Z.); mwang@phy.ecnu.edu.cn (M.W.); zwfang@phy.ecnu.edu.cn (Z.F.) \\ 3 XXL-The Extreme Optoelectromechanics Laboratory, School of Physics and Materials Science, \\ East China Normal University, Shanghai 200241, China \\ 4 School of Optoelectronics, University of Chinese Academy of Sciences, Beijing 100049, China \\ 5 Collaborative Innovation Center of Extreme Optics, Shanxi University, Taiyuan 030006, Shanxi, China \\ * Correspondence: ya.cheng@siom.ac.cn \\ + Jintian Lin and Junxia Zhou contributed equally to this work.
}

Received: 20 July 2019; Accepted: 14 September 2019; Published: 15 September 2019

\begin{abstract}
We demonstrate the fabrication of single-mode optical waveguides on lithium niobate on an insulator (LNOI) by optical patterning combined with chemomechanical polishing. The fabricated LNOI waveguides had a nearly symmetric mode profile of $\sim 2.5 \mu \mathrm{m}$ mode field size (full-width at half-maximum). We developed a high-precision measurement approach by which single-mode waveguides were characterized to have propagation loss of $\sim 0.042 \mathrm{~dB} / \mathrm{cm}$.
\end{abstract}

Keywords: lithium niobate; waveguide; photonic integrated circuit; optical lithography; chemomechanical polish

\section{Introduction}

Pursuing a photonic integrated circuit (PIC) has been taking place in the past few decades. inspired by the enormous success of electronic integration $[1,2]$. One of the key building blocks for realizing PICs is single-mode optical waveguides, by which the transportation and manipulation of photons can be realized in compact and stable optical networks. Major requirements for waveguides are low optical loss, and high tunability and nonlinearity. As an important platform for PICs, silicon-based optical waveguides provide high field confinement [3]. However, lack of second-order nonlinearity and electro-optic coefficients, and relatively high propagation loss are obstacles in silicon photonic applications. To this end, crystalline lithium niobate is almost an ideal candidate due to its broad transparent window, and high electro-optic and nonlinear coefficients $[4,5]$.

Early investigations in establishing PICs on lithium niobate (LN) focused on titanium-diffused LN waveguides with only subtle refractive index contrast, of which the large bending radius posed a scalability challenge [4]. On the other hand, several attempts were made on fabricating high-quality nanophotonic structures on lithium niobate on an insulator (LNOI) with either $\mathrm{Ar}^{+}$ion etching or diamond dicing. Results were not promising due to high surface roughness on the sidewalls of the fabricated structures [6,7]. The problem was tackled by employing focused-ion-beam (FIB) writing to realize a surface roughness of a few nanometers on the sidewalls of nanophotonic structures, resulting 
in LN microresonators with quality $(\mathrm{Q})$ factors on the order of $10^{6}$ [8-12]. Afterwards, combinations of either ultraviolet lithography or electron-beam lithography with reactive ion etching produced similar results on LNOI [12-16]. Nevertheless, nanometer-scale roughness left behind by dry ion etching was still an obstacle to further reduce the optical loss of the LNOI waveguide to below $10^{-1} \mathrm{~dB} / \mathrm{cm}$. In comparison with the intrinsic absorption loss of LN crystals, which is on the order of $10^{-3} \mathrm{~dB} / \mathrm{cm}$, the current optical loss could be improved by two orders of magnitude if scattering loss on the surface could be substantially eliminated. This provides a strong incentive to further suppress surface roughness on the sidewalls [17].

We recently developed a fabrication approach that allows to fabricate both high-Q factor LN microdisks and low-loss LN ridge waveguides [18-20]. The technique begins with femtosecond laser micromachining for patterning a hard chromium (Cr) mask coated on the LNOI, followed by chemomechanically polishing (CMP) the LNOI sample to transfer the generated pattern onto the LNOI. The fabricated waveguide in this manner showed surface roughness of $\mathrm{Rq} \sim 0.452 \mathrm{~nm}$ and propagation loss of $0.027 \mathrm{~dB} / \mathrm{cm}[19,20]$. Unfortunately, $\mathrm{LN}$ waveguides do not support single-mode propagation due to its relatively large cross-sectional dimensions, which is typically on the submicrometer scale but not $\sim 100 \mathrm{~nm}$ scale. Here, we converted the multimode $\mathrm{LN}$ waveguides to single-mode waveguides by covering them with a cladding layer, and examined the mode profiles and propagation losses in single-mode waveguides.

\section{Fabrication}

A commercially available X-cut LNOI wafer was used in the fabrication of the ridge waveguides. The LNOI wafer was produced by crystal ion slicing, and then bonded onto a silica layer of $2 \mu \mathrm{m}$ thickness supported by a bulk LN substrate of $500 \mu \mathrm{m}$ thickness. The structure of the LNOI wafer is shown in Figure 1a. The ridge waveguide was oriented along the $\mathrm{Y}$ axis. In such a configuration, the transverse-electric (TE) mode in the waveguide experiences the extraordinary refractive index of the LN crystal. The process flow for fabricating the ridge waveguides has five steps, as illustrated in Figure 1.

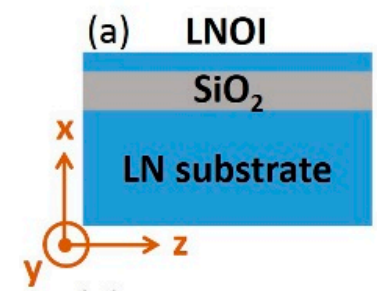

(d)

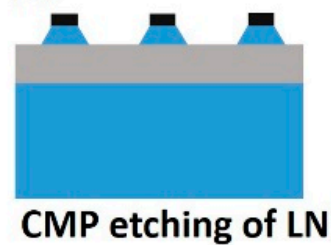

(b) Cr deposition

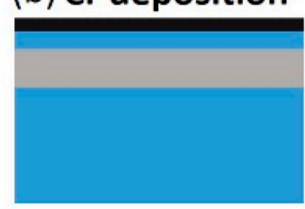

(e)

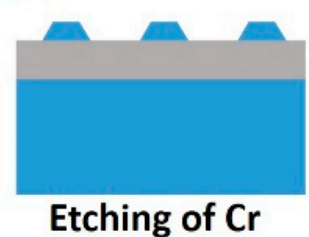

(c) Laser patterning

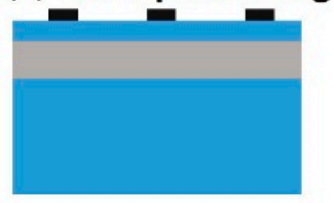

(f)

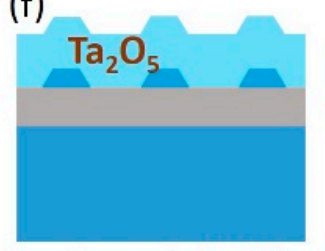

Coating of cladding

Figure 1. Process flow of lithium niobate on insulator (LNOI) waveguide fabrication. (a) Structure of LNOI substrate; (b) Depositing a layer of Cr film on LNOI; (c) Patterning the Cr film by femtosecond laser ablation for producing the mask of waveguides; (d) Transferring the mask pattern to LNOI by chemo-mechanical polish; (e) Removing the remaining $\mathrm{Cr}$ film by wet chemical etching; (f) Coating the waveguides with a layer of $\mathrm{Ta}_{2} \mathrm{O}_{5}$ to form the cladding.

First, a wear-resisting $\mathrm{Cr}$ layer with a thickness of $600 \mathrm{~nm}$ was coated onto the LNOI wafer by magnetron sputtering, as shown in Figure $1 \mathrm{~b}$. Note that $\mathrm{Cr}$ hardness was much higher than that of LN, making $\mathrm{Cr}$ good hard mask material for protecting the LN underneath in the CMP process. Second, the Cr layer was patterned into stripes with a width of $\sim 1 \mu \mathrm{m}$ by femtosecond laser ablation, 
as shown in Figure 1c. Femtosecond laser ablation was performed at a repetition rate of $250 \mathrm{kHz}$ and an average power of $0.05 \mathrm{~mW}$. An objective lens (Model: M Plan Apo NIR, Mitutoyo Corporation, Kanagawa, Japan) with a numerical aperture (NA) of 0.7 was used to focus the laser pulses, creating a focal spot of $1 \mu \mathrm{m}$ diameter on the sample. This objective lens was mounted onto a one-dimensional (1D) motion stage (Model: ANT130-110-L-ZS, Aerotech Inc., Hanover, MD, USA), which traveled in the vertical $(Z)$ direction at a resolution of $100 \mathrm{~nm}$ to ensure accurate focusing onto the sample surface. The sample was mounted onto an XY stage (Model: ABL15020WB and ABL15020, Aerotech Inc.) with a translation resolution of $100 \mathrm{~nm}$. The $\mathrm{Cr}$ was patterned by scanning the focal spot across the areas according the designed patterns. All motion stages were computer-programmable. The laser power we chose was sufficient for ablating $\mathrm{Cr}$, but insufficient for ablating the LN crystal, because the damage threshold of LN was higher than that of $\mathrm{Cr}$ [21]. This characteristic ensured that, in the ablation of $\mathrm{Cr}$, the LN thin film stayed intact. Afterwards, the LNOI wafer was subjected to CMP, which was carried out using a wafer-polishing machine. An LN ridge structure was obtained after $\mathrm{CMP}$, as shown in Figure 1d. A smooth sidewall with an average roughness of $\sim 0.5 \mathrm{~nm}$ was attainable by CMP. The $\mathrm{Cr}$ mask was removed via chemical etching by immersing the sample into a $\mathrm{Cr}$ etching solution for $4 \mathrm{~min}$, as shown in Figure 1e. Lastly, a layer of $\mathrm{Ta}_{2} \mathrm{O}_{5}$ was coated onto the sample to create a suitable refractive index contrast for ensuring single-mode waveguiding, as shown in Figure $1 \mathrm{f}$. The scanning-electrical-microscope (SEM) image of the fabricated LN ridge waveguide is presented in Figure 2a, and the waveguide cross-section is shown in the inset. Although the ablated resolution of $\mathrm{Cr}$ by femtosecond laser was on the magnitude of several tens of nanometers, the edge of the $\mathrm{Cr}$ stripes also endured CMP polishing. Therefore, this roughness did not transfer onto the LN ridge sidewall. The top width of the waveguide was determined to be $\sim 1.0 \mu \mathrm{m}$, whereas the bottom width was measured as $\sim 4.2 \mu \mathrm{m}$.
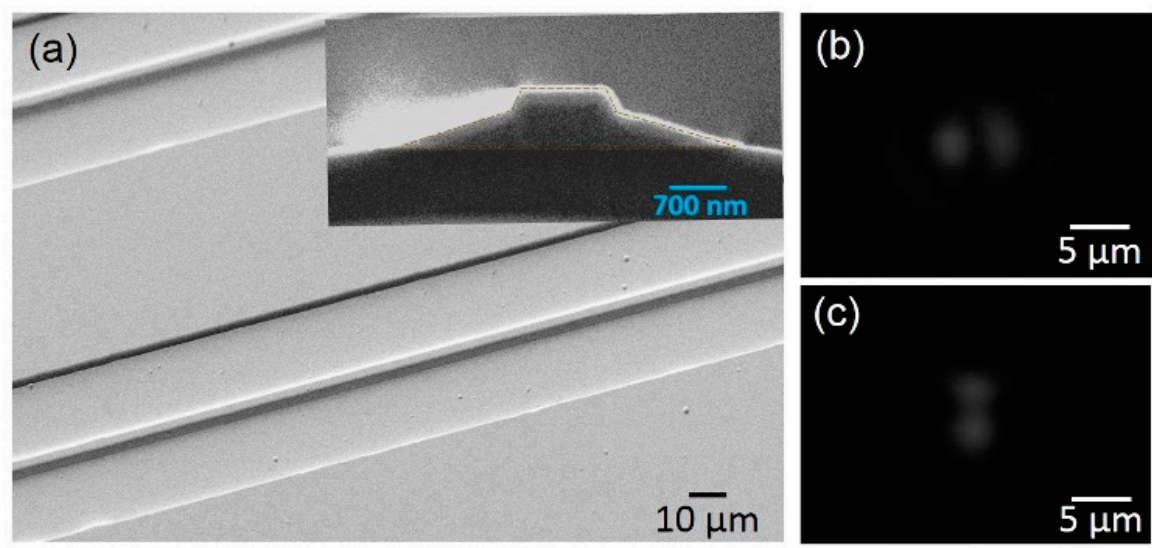

Figure 2. (a) Scanning-electron-microscope (SEM) images of lithium niobate (LN) waveguide before coating the $\mathrm{Ta}_{2} \mathrm{O}_{5}$ cladding layer) showing very smooth sidewalls. Inset: waveguide cross-section. Spatial distributions of (b) transverse-electric (TE) and (c) transverse-magnetic (TM) modes.

\section{Characterization}

Spatial modes were excited and characterized by coupling the waveguide with a $1550 \mathrm{~nm}$ wavelength laser using a fiber lens. The polarization state of the input light was controlled by an in-line polarization controller. The light transmitted from the output port of the waveguide was collected by an objective lens with $\mathrm{NA}=0.3$. A beam expander was introduced behind the objective lens, and it projected the image of the output port onto an infrared charge-coupled device (CCD) (InGaAs camera, HAMAMATSU Inc., Shizuoka, Japan). The spatial distributions of transverse-electric (TE) and transverse-magnetic (TM) modes were captured by the CCD, as shown in Figure 2b,c, respectively. The waveguide supported high-order spatial modes for the TE and TM modes owing to the high refractive index of LN and the large transverse dimensions of the waveguide. 
To produce single-mode LNOI waveguides for both the TE and the TM mode, $\mathrm{Ta}_{2} \mathrm{O}_{5}$ (refractive index 2.057) of ultralow loss [22] was deposited onto the fabricated sample by electron-beam evaporation. Figure 3a shows the scanning electron microscope (SEM) image of the LN ridge waveguide covered with the $\mathrm{Ta}_{2} \mathrm{O}_{5}$ cladding layer of $3.5 \mu \mathrm{m}$ thickness. A single-mode spatial profile was obtained for TE mode, as shown in Figure 3b, which was consistent with the calculated mode profile in Figure 3c. Similarly, such a waveguide supported single-mode propagation for TM mode as well, as shown in Figure $3 \mathrm{~d}$ and evidenced by the corresponding calculation result in Figure $3 \mathrm{e}$. The full width at half maximum (FWHM) of the TE mode was measured to be $\sim 2.5 \mu \mathrm{m}$, and the FWHM of the TM mode was $\sim 2.3 \mu \mathrm{m}$.

Traditionally, the propagation loss of a ridge waveguide can be measured based on the Fabry-Perot (FP) cavity-measurement or direct cut-back method [7,23-25]. However, ultralow loss of our chemomechanically polished LNOI waveguide could not be determined by the above methods due to insufficient precision. To overcome this difficulty, we developed a unique technique that allowed us to reliably measure nearly inappreciable loss in the on-chip LNOI waveguide.
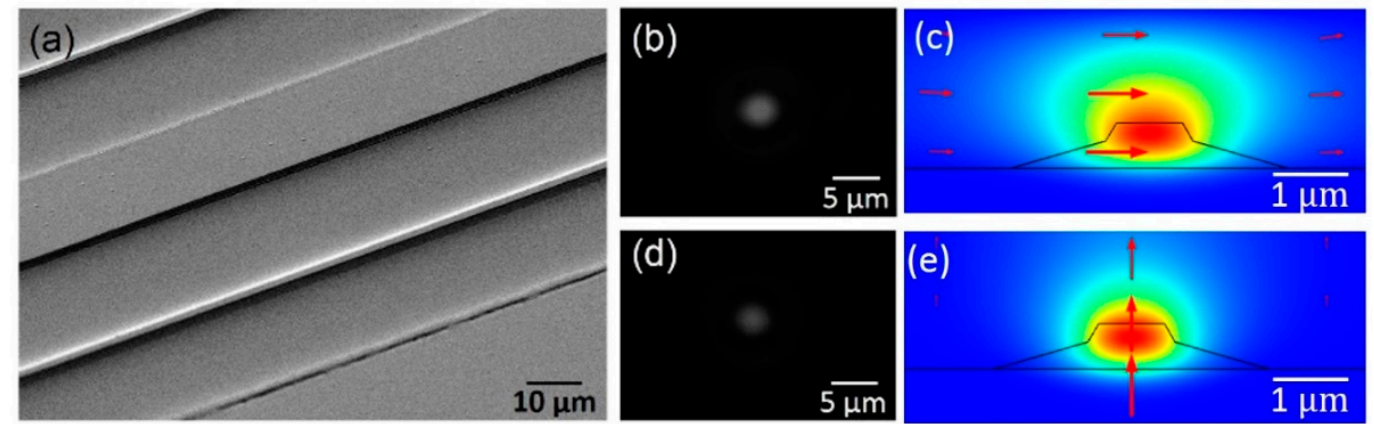

Figure 3. (a) SEM image of waveguide covered with $\mathrm{Ta}_{2} \mathrm{O}_{5}$. (b) Measured and (c) calculated TE mode profiles. (d) Measured and (e) calculated TM mode profiles.

Our high-precision loss-measurement method was established based on the design in Figure 4a. The device was composed of three beam splitters aligned in a vertical array, each of which consisting of two identical electro-optic (EO) Mach-Zehnder interferometers (MZIs) bridged by an EO phase shifter. A similar design was used to produce high-extinction-ratio beam splitters immune to fabrication imperfections [26,27]. The beam splitter was fabricated on an X-cut LNOI chip with its optic axis oriented perpendicularly to the MZI arms, as in Figure 4a. The beam-splitting ratio of the fabricated directional coupler (see Figure $4 \mathrm{~b}$ ) was designed to be 7:3. The output arms of different lengths were fabricated to differentiate propagation loss, i.e., one arm was $12 \mathrm{~mm}$ longer than the other. After fabrication of the LNOI waveguides, Au electrodes with a thickness of $200 \mathrm{~nm}$ were added by magnetron sputtering followed by space-selective patterning via femtosecond laser ablation, as shown in Figure 4c. Gap $d$ between the Au electrodes in each MZI was set as $10 \mu \mathrm{m}$, symmetrically arranging the two electrodes on both sides of the LNOI waveguides. The lengths of the interferometer arms of MZI 1, Phase Shifter, MZI 2, which were sandwiched between the Au electrodes, were 2, 11, and $2 \mathrm{~mm}$, respectively. A photograph of the fabricated device captured by a digital camera is shown in the inset of Figure $4 \mathrm{~b}$. The total length of the chip was $~ 30 \mathrm{~mm}$. 


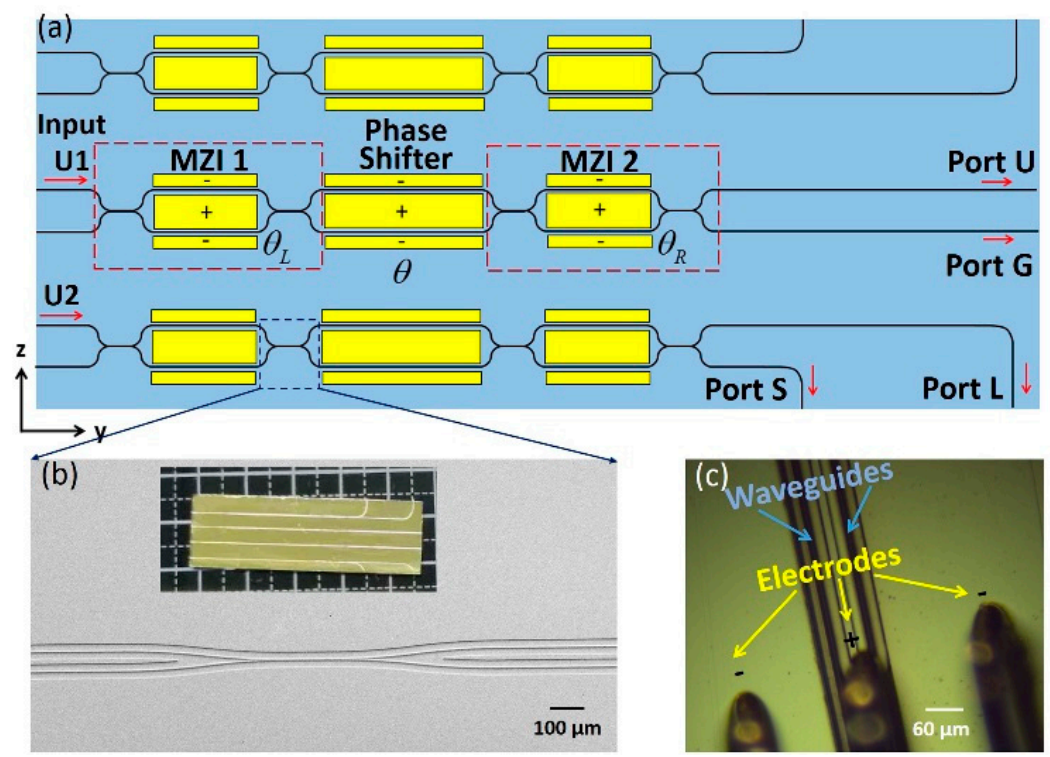

Figure 4. (a) Device layout. Here, phase differences $\theta_{\mathrm{L}}, \theta$, and $\theta_{\mathrm{R}}$ were contributed by fabrication imperfections of interferometer arms and of electro-optic (EO) modulation. (b) SEM image of directional coupler; inset: overview photograph of fabricated device consisting of three beam splitters.

(c) Optical-microscope image of electrodes contacted by three pins.

To characterize the EO response of the beam splitter, the telecom laser (New Focus Inc., San Jose, CA, USA, Model TLB 6728) with a pure TE mode at $1550 \mathrm{~nm}$ wavelength was coupled into input Port $\mathrm{U} 1$ of the beam splitter through a fiber lens. The output beam was first collimated by a $50 \times$ objective lens (Model: M Plan Apo NIR, Mitutoyo Corporation) of 0.42 NA, and then sent into an optical spectrum analyzer (OSA; YOKOGAWA Inc., Tamba City, Japan, Model AQ6370D, dynamic range $45 \mathrm{~dB}$ ). With this arrangement, the electric field was parallel to the optic axis of the $\mathrm{LN}$, as evidenced in Figure $5 \mathrm{a}$. Phase difference $\phi$ between the waves exiting from the two interferometer arms could be expressed as:

$$
\phi=\frac{2 \pi}{\lambda} n_{\mathrm{eff}}^{3} r_{33} \frac{V}{d} l
$$

Here, $\lambda$ is wavelength (i.e., $1550 \mathrm{~nm}), n_{\text {eff }}$ is the effective refractive index of $2.05, r_{33}\left(=30.8 \times 10^{-12} \mathrm{~m} / \mathrm{V}\right)$ is the largest electro-optic coefficient of $\mathrm{LN}$, and $V$ is the applied voltage. The minimal tuning step of the direct current voltage was set to $0.02 \mathrm{~V}$. Measured half-wave voltages $V_{\pi}$ were $6.7 \mathrm{~V}$ for the phase shifter and $36.8 \mathrm{~V}$ for MZIs 1 and 2. The results agreed well with the numerical calculation. Next, we tuned the splitting ratio for MZIs 1 and 2 to a precise 50:50 by using the following procedure [26]:

1. Adjust voltage on phase shifter to minimize the output power of output Port G.

2. Scan voltages on MZIs 1 and 2 to minimize the output power of Port G.

3. Repeat Steps 1 and 2, if necessary, until the minimal power in output $G$ is zero, and maximal power in Port $\mathrm{U}$ is as large as possible.

In our experiment, the extinction ratio between Ports $\mathrm{G}$ and $\mathrm{U}$ was determined to be $\sim 40 \mathrm{~dB}$ based on the experiment curve in Figure $5 \mathbf{b}$, which was obtained by varying phase difference $\theta$, giving rise to an oscillating power curve expressed by [26]:

$$
P_{U}=\frac{1}{2}(1-\cos \theta)
$$

The measured output power at Port $U$ in Figure $5 b$ nicely followed the cosine curve obtained using Equation (2), and that at Port G remained sinusoidal. 
(a)

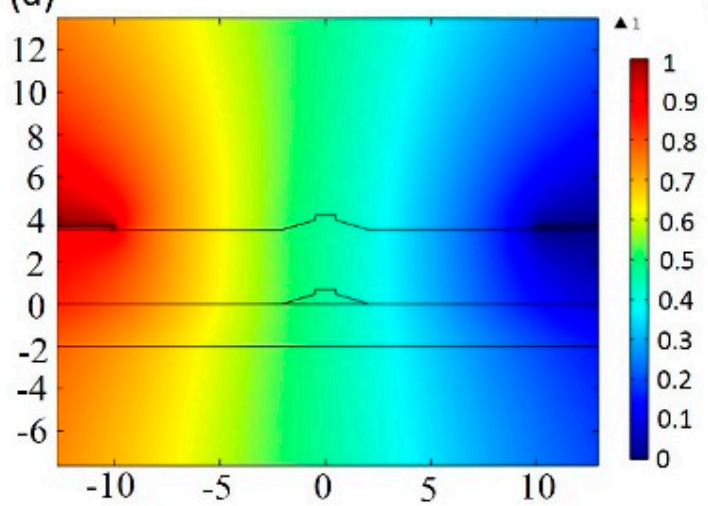

(b)

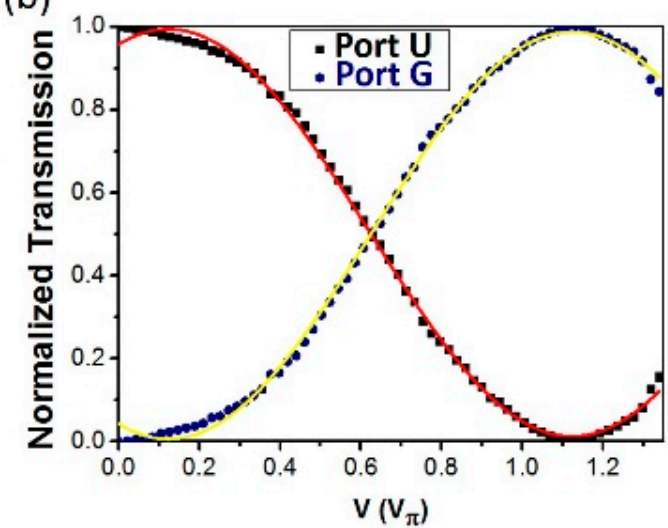

Figure 5. (a) Calculated normalized electric-field distribution in cross-sectional plane of LNOI waveguide when applying voltage on Au microelectrodes, showing that the electric field was almost parallel to the LN optic axis. (b) Normalized transmission spectra of output Ports U and G as a function of applied voltage $\mathrm{V}$, featuring sinusoidal-like curve.

To measure the propagation loss of the single-mode waveguide, the laser was coupled to input Port U2 of the bottom beam splitter in Figure 4a, and both MZIs were tuned at a 50:50 splitting ratio via EO modulation. Then, power output from Port $S$ was tuned to be maximal by adjusting the voltage applied on the phase shifter. Finally, power output from Port $S$ was tuned to be half the maximal value. In other words, phase difference $\theta$ between the two arms of the phase shifter in the bottom beam splitter was $\pi / 2$, and the powers injected into output Ports $S$ and $L$ were the same. Propagation loss was measured to be $0.042 \pm 0.02 \mathrm{~dB} / \mathrm{cm}$ by comparing the powers at output Ports $S$ (averaged counts: 3943) and L (averaged counts: 3898). Median measured error was originated from the minimal tuning step of the voltage source.

\section{Conclusions}

To conclude, we fabricated single-mode LNOI waveguides with a propagation loss of $\sim 0.042 \mathrm{~dB} / \mathrm{cm}$ and a mode field size of $\sim 2.5 \mu \mathrm{m}$. High-precision loss measurement was achieved using an EO controllable beam splitter to ensure the simultaneous injection of two light waves of the same input power into two waveguides of unbalanced lengths. This method avoids fluctuations in coupling efficiency when carrying conventional cut-back measurement, enabling the differentiation of two waveguides of propagation losses close to each other. Low-loss single-mode LN waveguides can be used for constructing complex photonic circuits.

Author Contributions: Conceptualization, Y.C.; methodology, Y.C., J.L., and J.Z. (Junxia Zhou); software, J.L. and M.W.; validation, J.L.; formal analysis, J.L. and J.Z. (Junxia Zhou); investigation, J.L. and J.Z. (Junxia Zhou); resources, J.L., J.Z. (Jianhao Zhang), R.W., M.W., W.C., Z.F., and L.Q.; data curation, J.L. and J.Z. (Junxia Zhou); writing-original-draft preparation, Y.C. and J. L.; writing-review and editing, Y.C. and J.L.; visualization, J.L.; supervision, Y.C.; funding acquisition, Y.C., J.L., and L.Q.

Funding: This research was funded by the National Natural Science Foundation of China (Grant Nos. 11734009, 11874375, 61590934, 11874154, 61505231, 11604351, 11674340, 61575211, 61675220, 61761136006); the Key Research Program of Frontier Sciences, CAS (Grant No. QYZDJ-SSW-SLH010); the Key Project of the Shanghai Science and Technology Committee (Grant Nos. 18DZ1112700 and 17JC1400400); and the Strategic Priority Research Program of the Chinese Academy of Sciences (XDB16030300).

Conflicts of Interest: The authors declare no conflict of interest. 


\section{References}

1. Brenner, K.H.; Huang, A. Logic and architectures for digital optical computers. J. Opt. Soc. Am. A 1986, 3, 62.

2. Boes, A.; Corcoran, B.; Chang, L.; Bowers, J.; Mitchell, A. Status and potential of lithium niobate on insulator (LNOI) for photonic integrated circuits. Laser Photon. Rev. 2018, 12, 1700256. [CrossRef]

3. Chrostowski, L.; Hochberg, M. Silicon Photonics Design; Cambridge University Press: Cambridge, UK.

4. Jin, H.; Liu, F.M.; Xu, P.; Xia, J.L.; Zhong, M.L.; Yuan, Y.; Zhou, J.W.; Gong, Y.X.; Wang, W.; Zhu, S.N. On-chip generation and manipulation of entangled photons based on reconfigurable lithium-niobate waveguide circuits. Phys. Rev. Lett. 2014, 113, 103601. [CrossRef] [PubMed]

5. Kösters, M.; Sturman, B.; Werheit, P.; Haertle, D.; Buse, K. Optical cleaning of congruent lithium niobate crystals. Nat. Photon. 2009, 3, 510-513. [CrossRef]

6. Guarino, A.; Poberaj, G.; Rezzonico, D.; Degl'Innocenti, R.; Günter, P. Electro-optically tunable microring resonators in lithium niobate. Nat. Photon. 2007, 1, 407-410. [CrossRef]

7. Volk, M.F.; Suntsov, S.; Rüter, C.E.; Kip, D. Low loss ridge waveguides in lithium niobate thin films by optical grade diamond blade dicing. Opt. Express 2016, 24, 1386-1391. [CrossRef] [PubMed]

8. Lin, J.; Xu, Y.; Fang, Z.; Wang, M.; Song, J.; Wang, N.; Qiao, L.; Fang, W.; Cheng, Y. Second harmonic generation in a high-Q lithium niobate microresonator fabricated by femtosecond laser micromachining. Sci. China Phys. Mech. Astrono. 2015, 58, 114209. [CrossRef]

9. Lin, J.; Xu, Y.; Fang, Z.; Wang, M.; Song, J.; Wang, N.; Qiao, L.; Fang, W.; Cheng, Y. Fabrication of high-Q lithium niobate microresonators using femtosecond laser micromachining. Sci. Rep. 2015, 5, 8072. [CrossRef]

10. Liu, S.; Zheng, Y.; Chen, X. Cascading second-order nonlinear processes in a lithium niobate-on-insulator microdisk. Opt. Lett. 2017, 42, 3626-3629. [CrossRef]

11. Lin, J.; Yao, N.; Hao, Z.; Zhang, J.; Mao, W.; Wang, M.; Chu, W.; Wu, R.; Fang, Z.; Qiao, L.; et al. Broadband quasi-phase-matched harmonic generation in an on-chip monocrystalline lithium niobate microdisk resonator. Phys. Rev. Lett. 2019, 122, 173903. [CrossRef]

12. Lin, J.; Xu, Y.; Ni, J.; Wang, M.; Fang, Z.; Qiao, L.; Fang, W.; Cheng, Y. Phase-matched second-harmonic generation in an on-chip LiNbO3.microresonator. Phys. Rev. Appl. 2016, 6, 014002. [CrossRef]

13. Wang, J.; Bo, F.; Wan, S.; Li, W.; Gao, F.; Li, J.; Zhang, G.; Xu, J. High-Q lithium niobate microdisk resonators on a chip for efficient electro-optic modulation. Opt. Express 2015, 23, 23072-23078. [CrossRef] [PubMed]

14. Wang, L.; Wang, C.; Wang, J.; Bo, F.; Zhang, M.; Gong, Q.; Lončar, M.; Xiao, Y.-F. High-Q chaotic lithium niobate microdisk cavity. Opt. Lett. 2018, 43, 2917-2920. [CrossRef] [PubMed]

15. Zhang, M.; Wang, C.; Cheng, R.; Shams-Ansari, A.; Lončar, M. Monolithic ultra-high-Q lithium niobate microring resonator. Optica 2017, 4, 1536-1537. [CrossRef]

16. Luo, R.; Jiang, H.; Rogers, S.; Liang, H.; He, Y.; Lin, Q. On-chip second-harmonic generation and broadband parametric down-conversion in a lithium niobate microresonator. Opt. Express 2017, 25, 24531-24539. [CrossRef] [PubMed]

17. Wolf, R.; Breunig, I.; Zappe, H.; Buse, K. Cascaded second-order optical nonlinearities in on-chip micro rings. Opt. Express 2017, 25, 29927-29933. [CrossRef] [PubMed]

18. Wu, R.; Zhang, J.; Yao, N.; Fang, W.; Qiao, L.; Chai, Z.; Lin, J.; Cheng, Y. Lithium niobate micro-disk resonators of quality factors above $10^{7}$. Opt. Lett. 2018, 43, 4116-4119. [CrossRef] [PubMed]

19. Wu, R.; Wang, M.; Xu, J.; Qi, J.; Chu, W.; Fang, Z.; Zhang, J.; Zhou, J.; Qiao, L.; Chai, Z.; et al. Long low-loss-litium niobate on insulator waveguides with sub-nanometer surface roughness. Nanomaterials 2018, 8,910. [CrossRef]

20. Wang, M.; Wu, R.; Lin, J.; Zhang, J.; Fang, Z.; Chai, Z.; Cheng, Y. Chemo-mechanical polish lithography: A pathway to low loss large-scale photonic integration on lithium niobate on insulator. Quant. Eng. 2019, 1, e9. [CrossRef]

21. Joglekar, A.P.; Liu, H.-H.; Meyhöfer, E.; Mourou, G.; Hunt, A.J. Optics at critical intensity: Applications to nanomorphing. Proc. Natl. Acad. Sci. USA 2004, 101, 5856-5861. [CrossRef]

22. Belt, M.; Davenport, M.L.; Bowers, J.E.; Blumenthal, D.J. Ultra-low-loss $\mathrm{Ta}_{2} \mathrm{O}_{5}-\mathrm{core} / \mathrm{SiO}_{2}$-clad planar waveguides on Si substrates. Optica 2017, 4, 532-536. [CrossRef]

23. Regener, R.; Sohler, W. Loss in low-finesse Ti: $\mathrm{LiNbO}_{3}$ optical waveguide resonators. Appl. Phys. B 1985, 36, 143-147. [CrossRef]

24. Walker, R.G. Simple and accurate loss measurement technique for semiconductor optical waveguide. Electron. Lett. 1985, 21, 581-583. [CrossRef] 
25. Hunsperger, R.G. Integrated Optics: Theory and Technology, 3rd ed.; Springer: New York, NY, USA, 1991.

26. Miller, D.A.B. Perfect optics with imperfect components. Optica 2015, 2, 747-750. [CrossRef]

27. Jin, M.; Chen, J.-Y.; Sua, Y.M.; Huang, Y.-P. High-extinction electro-optic modulation on lithium niobate thin film. Opt. Lett. 2019, 44, 1265-1268. [CrossRef] [PubMed]

(C) 2019 by the authors. Licensee MDPI, Basel, Switzerland. This article is an open access article distributed under the terms and conditions of the Creative Commons Attribution (CC BY) license (http://creativecommons.org/licenses/by/4.0/). 